\title{
The Potential of Glioblastoma Patient Symptoms to Diagnose and Predict Survival
}

\author{
Oliver D. Mrowczynski ${ }^{1}$, Ae L. Yang ${ }^{1}$, Jiangang Liao ${ }^{2}$, Elias Rizk ${ }^{3}$ \\ 1. Neurosurgery, Penn State Health Milton S. Hershey Medical Center, Hershey, USA 2. Public Health Sciences, Penn \\ State Health Milton S. Hershey Medical Center, Hershey, USA 3. Neurological Surgery, Penn State Health Milton S. \\ Hershey Medical Center, Hershey, USA
}

Corresponding author: Oliver D. Mrowczynski, omrowczynski@pennstatehealth.psu.edu

\begin{abstract}
Glioblastoma is a devastating malignancy with a dismal survival rate and median survival time of 14 months. Currently, the biomarkers for glioblastoma are mostly molecular and include EGFRvIII, ATRX, PTEN, IDH1, MGMT, and others. These prognostic tumor biomarkers are obtained through a surgical biopsy and thus are not easily attainable. Clinicians would benefit from a robust, non-invasive, and readily available indicator for early diagnosis and accurate prognostication for glioblastoma patients. In this study, we assessed whether specific patient symptoms could provide an early diagnosis of glioblastoma. Further, we also assessed if any patient symptomatology could provide clinicians with the ability to prognosticate patient survival more accurately. We retrospectively reviewed the clinical data for 218 patients. We determined whether symptoms including headache, weakness, seizure, memory loss/confusion, visual changes, speech changes, and loss of consciousness led to a patient being diagnosed earlier and if any of these symptoms predicted diminished patient survival. Our study determined that weakness and memory loss/confusion were the symptoms that predicted diminished survival, and weakness alone was the symptom that predicted an earlier diagnosis. This study further elucidates the complexities of glioblastoma and provides clinicians with more data for their patients when discussing prognostication after diagnosis of glioblastoma.
\end{abstract}

Categories: Neurosurgery

Keywords: glioblastoma, glioma, symptoms, diagnosis, prognosis, survival

\section{Introduction}

Glioblastoma is a malignant brain tumor in adults with a dismal survival rate and median survival time of 14 months [1]. Glioblastoma, unfortunately, affects five per 100,000 people, making it the most common malignant brain tumor in adults [2]. Currently, the standard protocol for treatment includes maximal safe surgical resection with adjuvant chemotherapy of temozolomide (Temodar, MERCK \& CO., Inc, Whitehouse Station, NJ) and radiation in large doses [3]. The current prognostic markers for glioblastoma are molecular and are obtained through a surgical biopsy. These biomarkers include EGFRvIII, ATRX, PTEN, IDH1, MGMT, and others [1,3-9]. Some are also currently used as markers to prognosticate patients and predict response to therapies $[10,11]$. Markers that are easily clinically attainable and can provide a robust prognostic indicator and early diagnosis would be beneficial for patients suffering from glioblastoma.

Received 05/26/2021

eview began 06/09/2021 Published 07/27/2021

๑) Copyright 2021

Mrowczynski et al. This is an open access article distributed under the terms of the Creative Commons Attribution License CC-BY 4.0., which permits unrestricted use, distribution, and reproduction in any medium, provided the original author and source are credited.
This study assesses whether symptoms including headache, weakness, seizure, memory loss/confusion, visual changes, speech changes, and loss of consciousness lead to a patient being diagnosed earlier and if any of these symptoms can predict diminished patient survival. Patient's clinical exam is easily attainable, thus making it an optimal marker. Understanding the relationship between glioblastoma patient symptomatology and diagnosis and prognosis may pave the way to utilizing symptoms as a robust and quick factor for patient prognostication. It also provides clinicians with more data to use when discussing with patients their prognosis and more accurately provide information about their disease.

In this study, we retrospectively analyze glioblastoma patients treated at our institution, comparing the time of diagnosis and patient survival depending on their specific symptomatology. Specifically, this study analyzes whether specific symptoms can predict early diagnosis or predict a decreased survival. We further aim to elucidate the complexities of this devastating disease. This study helps determine the underlying nuances of glioblastoma. It provides clinicians more data to speak to patients when discussing prognostication after a diagnosis of glioblastoma.

This article was previously published as a preprint at https://www.researchsquare.com/article/rs200717/v1 as Clinical Study: Mrowczynski O, Yang AL, Liao J, Langan S, Rizk E. The Predictive and Diagnostic Potential of Symptoms for Glioblastoma Patient Survival. DOI: 10.21203/rs.3.rs-200717/v1.

\section{Materials And Methods}




\section{Cureus}

\section{Patient population}

A retrospective chart analysis that included 218 histopathology-confirmed and diagnosed glioblastoma patients at the Pennsylvania State University Department of Neurosurgery from 2006 until 2016 was included in the study. The patients in this study were all pathologically confirmed to have WHO grade-IV glioblastoma multiforme. Most patients had the standard protocol for glioblastoma treatment, including maximal safe surgical resection, chemotherapy with temozolomide, and high-dose 60Gy radiation. Patients who had a previous low-grade glioma and any therapeutic intervention for a previous low-grade glioma were excluded from the study. Patients whose date of death could not be accurately determined were excluded.

\section{Data collection and statistical analysis}

The dataset consisted of 218 subjects. Days from the first symptom to death, days from the first symptom to diagnosis, and days from the diagnosis to death were calculated for each subject. The dependence of these three time-to-event variables on various predictors was modeled using Cox's proportional hazards models. These predictors included age; sex; symptoms including headache, weakness, seizure, memory loss and confusion, visual changes, speech changes, loss of consciousness; and treatments, including resection, radiation, and Temodar. Additionally, days from the first symptom to diagnosis is also used as a potential predictor for days from diagnosis to death. Specifically, this study analyzes whether specific symptoms can predict an early diagnosis or decreased survival. All data in this study were subjected to statistical analysis. Our final Cox model for each time-to-event variable consists of only statistically significant predictors. The analysis was conducted using R 3.4 (R Foundation for Statistical Computing). A p-value $<0.05$ was deemed statistically significant. This study received Penn State Health Milton S. Hershey Medical Center Institutional Review Board approval, IRB \#5691.

\section{Results}

\section{Patient characteristics}

The characteristics of our 218 patients are shown in Table 1 .

\begin{tabular}{|c|c|}
\hline & $N(\%)$ \\
\hline Median age, years (range) & $64(5-88)$ \\
\hline \multicolumn{2}{|l|}{ Sex: } \\
\hline Male & $110(51)$ \\
\hline Female & $108(49)$ \\
\hline \multicolumn{2}{|l|}{ Symptoms: } \\
\hline Asymptomatic & $4(1.8)$ \\
\hline Headache & $65(29.8)$ \\
\hline Weakness & $77(35.3)$ \\
\hline Seizure & $21(9.6)$ \\
\hline Memory loss/Confusion & $86(39.5)$ \\
\hline Visual Changes & $15(6.9)$ \\
\hline Speech Changes & $55(25.2)$ \\
\hline Loss of Consciousness & $4(1.8)$ \\
\hline \multicolumn{2}{|l|}{ Treatments: } \\
\hline Resection & $135(61.9)$ \\
\hline Radiation & $148(67.9)$ \\
\hline Temodar & $152(69.7)$ \\
\hline
\end{tabular}

TABLE 1: Patient Characteristics ( $\mathrm{N}=\mathbf{2 1 8})$ 


\section{Cureus}

male, comprising $51 \%$ of our sample population, while 108 patients were female, comprising $49 \%$ of our sample population. We also described the number of patients with specific symptoms; some patients had multiple presenting symptoms. Four patients were asymptomatic, comprising $1.8 \%$ of our sample population. Sixty-five patients had a headache, comprising $29.8 \%$ of our sample population. Seventy-seven presented with weakness, comprising $35.3 \%$ of our sample population. Twenty-one had a seizure, comprising $9.6 \%$ of the population. Eighty-six had memory loss/confusion, comprising $39.5 \%$ of our sample population. Fifteen patients had visual changes, comprising $6.9 \%$ of our sample population. Fifty-five had speech changes, comprising $25.2 \%$ of our sample population. Four had a loss of consciousness, comprising $1.8 \%$ of our sample population. We also analyzed the data of the patients in our sample who were treated. One hundred and thirty-five patients had a surgical resection, comprising $61.9 \%$ of our sample population. One hundred and forty-eight received radiation, comprising $67.9 \%$ of our sample population, and 152 took Temodar, comprising $69.7 \%$ of our sample population.

\section{Symptom prediction of survival}

Patient symptoms as a predictor of survival is shown in Table 2 .

\begin{tabular}{|c|c|c|}
\hline & P Value & Hazard Ratio and $95 \%$ Confidence Interval \\
\hline Age & $3.17 \times 10^{\wedge}-5$ & 1.027 [1.014-1.039] \\
\hline Sex (male) & 0.253 & 1.191 [0.883-1.607] \\
\hline \multicolumn{3}{|l|}{ Symptoms: } \\
\hline Headache & 0.239 & $0.821[0.592-1.14]$ \\
\hline Weakness & 0.0063 & $1.543[1.131-2.106]$ \\
\hline Seizure & 0.206 & 0.717 [0.427-1.201] \\
\hline Memory loss/Confusion & 0.0588 & $1.345[0.989-1.83]$ \\
\hline Visual Changes & 0.21 & $0.651[0.332-1.274]$ \\
\hline Speech Changes & 0.642 & 1.084 [0.771-1.524] \\
\hline Loss of Consciousness & 0.626 & $0.707[0.175-2.855]$ \\
\hline \multicolumn{3}{|l|}{ Treatments: } \\
\hline Resection & 0.00085 & $0.593[0.436-0.806]$ \\
\hline Radiation & $1.06 \times 10^{\wedge}-10$ & $0.338[0.243-0.47]$ \\
\hline Temodar & $<2 \times 10^{\wedge}-16$ & $0.222[0.157-0.314]$ \\
\hline
\end{tabular}

TABLE 2: Predictor of Survival

The older the patient, the greater the decrease in survival $\left(\mathrm{P}=3.17 \times 10^{\wedge}-5\right.$, hazard ratio [HR] 1.027 [1.0141.039]). Patients presenting with weakness had a decreased survival, which was statistically significant $(\mathrm{P}=0.0063 ; \mathrm{HR} 1.543$ [1.131-2.106]). Patients presenting with memory loss/confusion had a decreased survival, which trended toward statistical significance ( $\mathrm{P}=0.0588$; HR 1.345 [0.989-1.83]). All other symptoms, including headache, seizure, visual changes, speech changes, and loss of consciousness, did not predict survival. As a control for our study, we evaluated the predictor of survival when patients received treatment with resection, radiation, and/or Temodar. Patients who had a resection had an increased survival $(\mathrm{P}=0.00085$; HR 0.593 [0.436-0.806]). Patients who had radiation and Temodar also had an increased survival $\left(\mathrm{P}=1.06 \times 10^{\wedge}-10\right.$; HR 0.338 [0.243-0.47] and $\mathrm{P}=<2 \times 10^{\wedge}-16$; HR 0.222 [0.157-0.314], respectively). We also looked at the effects of resection and weakness. Figure $1 \mathrm{~A}$ shows that not having a resection led to a lower survival probability $(\mathrm{P}=0.04)$. Figure $1 B$ shows that patients with weakness in the group of patients who did have a resection tended toward a shorter and decreased survival probability $(\mathrm{P}=0.08)$. 


\section{Cureus}
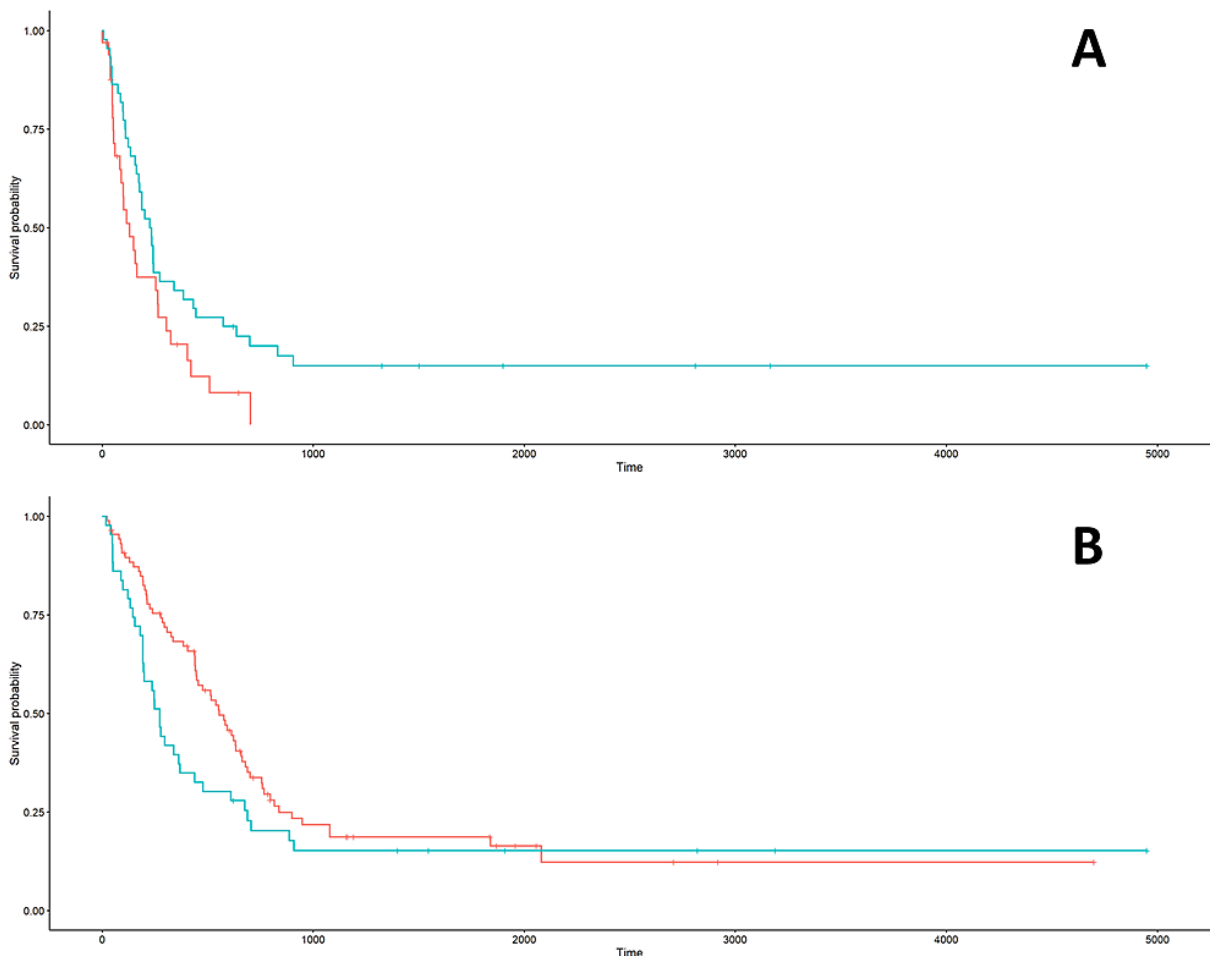

FIGURE 1: Effects of Weakness and Resection on Survival

(1A) In the group of patients who had a weakness, not having a resection led to a decreased survival $(P=0.04)$. (1B) In the group of patients who did have a resection, patients with weakness trended toward a shorter and decreased survival $(P=0.08)$.

\section{Symptom prediction of diagnosis}

Patient symptoms as a predictor of early diagnosis are shown in Table 3.

\begin{tabular}{|c|c|c|}
\hline & P Value & Hazard Ratio and $95 \%$ Confidence Interval \\
\hline Age & 0.625 & $0.998[0.988-1.008[$ \\
\hline Sex (male) & 0.235 & $1.18[0.898-1.551]$ \\
\hline \multicolumn{3}{|l|}{ Symptoms: } \\
\hline Headache & 0.62 & $1.077[0.804-1.444]$ \\
\hline Weakness & 0.0152 & 1.428 [1.071-1.904] \\
\hline Seizure & 0.891 & $1.0326[0.657-1.622]$ \\
\hline Memory loss/Confusion & 0.344 & $0.875[0.664-1.154]$ \\
\hline Visual Changes & 0.72 & 0.905 [0.526-1.559] \\
\hline Speech Changes & 0.636 & $0.927[0.679-1.266]$ \\
\hline Loss of Consciousness & 0.151 & $0.483[0.1 / 84-1.305]$ \\
\hline
\end{tabular}

TABLE 3: Predictor of Diagnosis

Age and sex were not found to be predictors of an earlier diagnosis. Patients who presented with weakness as a symptom had a statistically significant increase in their speed of diagnosis $(\mathrm{P}=0.0152$; HR 1.428 [1.071-

1.904]). All other symptoms, including headache, seizure, memory loss/confusion, visual changes, speech changes, and loss of consciousness, did not predict an earlier diagnosis. 


\section{Discussion}

Glioblastoma patients have a devastating prognosis, and non-invasive patient prognosis markers are important to help patients understand their disease and for the physician to more accurately predict a patient's survival. The patient's symptoms are one of the most easily attainable factors of a glioblastoma patient presentation. The possibility of using these data to prognosticate better a patient's clinical course is something to be desired. The better characterization of a glioblastoma patient regarding symptomatology also helps to understand this complex disease further. These data help the clinician be more accurate and personalized when discussing a patient's prognosis and disease course. To our knowledge, this is the first study analyzing and characterizing symptoms with regard to glioblastoma patient diagnosis and prognosis.

Our data included controls, e.g., the patient's age and treatment with surgical resection and adjuvant chemoradiation. As is known, older patients have diminished survival, as shown in our data,. Additionally, surgical resection and treatment with adjuvant chemotherapy and radiation are known to increase survival, which is also shown in our data. These factors were used to demonstrate our patient cohort following the known trends in the literature.

As far as weakness is concerned as a symptom that predicted earlier diagnosis, this may be because it is easily recognizable by the patient or their family. A patient's family that lives with them is able to realize that something is wrong with their family member and brings them to the emergency department to be evaluated. Indeed, multiple other studies in different cancer types including prostate, breast, multiple myeloma, elderly glioma, colorectal, and head and neck cancers have looked at weakness and frailty as a predictor of patient survival [12-18]. These studies show that weakness is a predictor of poor outcome in cancer. Furthermore, studies have shown that cognitive deterioration precedes MRI progression [19]. Symptoms like visual changes may not bring the patient to be evaluated sooner as the patient may be in denial of the occurring changes. These findings are different than the Karnofsky Performance Scale (KPS) as KPS is a functional impairment scale and not specific to weakness as a presentation. As far as weakness and memory loss/confusion being a predictor for decreased survival is concerned, this may be because these symptoms cause significant decrease in daily functions. Previous studies have also looked at the test of verbal memory in glioma patients and found that verbal memory was independently and strongly associated with survival [20]. The patient being unable to care for themselves in the same way, which as before may lead to decreased patient survival overall. We show that in the group of patients who did have a resection, weakness was an independent predictor of poor outcome.

This study's limitations include its retrospective nature and that it only assesses the outcomes of patients in one institution. It also did not take into account a patient's financial status, which may have an impact on the availability of treatments and, thus, patient survival.

\section{Conclusions}

This study demonstrates that weakness and memory loss/confusion were the symptoms that predicted diminished survival, and weakness alone was the symptom that predicted an earlier diagnosis. This study further helps to elucidate the complexities of glioblastoma and provides clinicians with more data which they can provide to patients when discussing prognostication after a diagnosis of glioblastoma. Further studies must be performed in larger cohorts in other institutions to confirm this finding further.

\section{Additional Information \\ Disclosures}

Human subjects: Consent was obtained or waived by all participants in this study. Penn State Health Milton S. Hershey Medical Center Institutional Review Board issued approval IRB \#5691. Animal subjects: All authors have confirmed that this study did not involve animal subjects or tissue. Conflicts of interest: In compliance with the ICMJE uniform disclosure form, all authors declare the following: Payment/services info: All authors have declared that no financial support was received from any organization for the submitted work. Financial relationships: All authors have declared that they have no financial relationships at present or within the previous three years with any organizations that might have an interest in the submitted work. Other relationships: All authors have declared that there are no other relationships or activities that could appear to have influenced the submitted work.

\section{Acknowledgements}

We would like to thank Sara Langan for her editorial assistance.

\section{References}

1. Stupp R, Mason WP, van den Bent MJ, et al.: Radiotherapy plus concomitant and adjuvant temozolomide for glioblastoma. N Engl J Med. 2005, 352:987-96. 10.1056/NEJMoa043330

2. Wen PY, Kesari S: Malignant gliomas in adults. N Engl J Med. 2008, 359:492-507. 10.1056/NEJMra0708126

3. Stupp R, Hegi ME, Mason WP, et al.: Effects of radiotherapy with concomitant and adjuvant temozolomide 
versus radiotherapy alone on survival in glioblastoma in a randomised phase III study: 5-year analysis of the EORTC-NCIC trial. Lancet Oncol. 2009, 10:459-66. 10.1016/S1470-2045(09)70025-7

4. Liu F, Hon GC, Villa GR, et al.: EGFR mutation promotes glioblastoma through epigenome and transcription factor network remodeling. Mol Cell. 2015, 60:307-18. 10.1016/j.molcel.2015.09.002

5. van den Bent MJ, Gao Y, Kerkhof M, et al.: Changes in the EGFR amplification and EGFRvIII expression between paired primary and recurrent glioblastomas. Neuro Oncol. 2015, 17:935-41. 10.1093/neuonc/nov013

6. Xu J, Li Z, Wang J, Chen H, Fang JY: Combined PTEN mutation and protein expression associate with overall and disease-free survival of glioblastoma patients. Transl Oncol. 2014, 7:196-205.e1. 10.1016/j.tranon.2014.02.004

7. Ueki K, Ono Y, Henson JW, Efird JT, von Deimling A, Louis DN: CDKN2/p16 or RB alterations occur in the majority of glioblastomas and are inversely correlated. Cancer Res . 1996, 56:150-3.

8. Sturm D, Witt H, Hovestadt V, et al.: Hotspot mutations in H3F3A and IDH1 define distinct epigenetic and biological subgroups of glioblastoma. Cancer Cell. 2012, 22:425-37. 10.1016/j.ccr.2012.08.024

9. Chaurasia A, Park SH, Seo JW, Park CK: Immunohistochemical analysis of ATRX, IDH1 and p53 in glioblastoma and their correlations with patient survival. J Korean Med Sci. 2016, 31:1208-14. 10.3346/jkms.2016.31.8.1208

10. Kleihues P, Ohgaki H: Primary and secondary glioblastomas: from concept to clinical diagnosis . Neuro Oncol. 1999, 1:44-51. 10.1093/neuonc/1.1.44

11. Ohgaki H, Kleihues P: The definition of primary and secondary glioblastoma. Clin Cancer Res. 2013, 19:76472. 10.1158/1078-0432.CCR-12-3002

12. Cloney M, D'Amico R, Lebovic J, et al.: Frailty in geriatric glioblastoma patients: a predictor of operative morbidity and outcome. World Neurosurg. 2016, 89:362-7. 10.1016/j.wneu.2015.12.096

13. Gao ZY, Zhang T, Zhang H, Pang CG, Jiang WX: Prognostic factors for overall survival in patients with spinal metastasis secondary to prostate cancer: a systematic review and meta-analysis. BMC Musculoskelet Disord. 2020, 21:388. 10.1186/s12891-020-03412-0

14. Ommundsen N, Wyller TB, Nesbakken A, Jordhøy MS, Bakka A, Skovlund E, Rostoft S: Frailty is an independent predictor of survival in older patients with colorectal cancer. Oncologist. 2014, 19:1268-75. 10.1634/theoncologist.2014-0237

15. Zakaria HM, Basheer A, Boyce-Fappiano D, et al.: Application of morphometric analysis to patients with lung cancer metastasis to the spine: a clinical study. Neurosurg Focus. 2016, 41:E12. 10.3171/2016.5.FOCUS16152

16. Zakaria HM, Llaniguez JT, Telemi E, et al.: Sarcopenia predicts overall survival in patients with lung, breast, prostate, or myeloma spine metastases undergoing stereotactic body radiation therapy (SBRT), independent of histology. Neurosurgery. 2020, 86:705-16. 10.1093/neuros/nyz216

17. Zakaria HM, Massie L, Basheer A, et al.: Application of morphometrics as a predictor for survival in female patients with breast cancer spinal metastasis: a retrospective cohort study. Spine J. 2018, 18:1798-803. 10.1016/j.spinee.2018.03.007

18. Zakaria HM, Massie L, Basheer A, et al.: Application of morphometrics as a predictor for survival in patients with prostate cancer metastasis to the spine. World Neurosurg. 2018, 114:e913-9. 10.1016/j.wneu.2018.03.115

19. Meyers CA, Hess KR: Multifaceted end points in brain tumor clinical trials: cognitive deterioration precedes MRI progression. Neuro Oncol. 2003, 5:89-95. 10.1093/neuonc/5.2.89

20. Meyers CA, Hess KR, Yung WK, Levin VA: Cognitive function as a predictor of survival in patients with recurrent malignant glioma. J Clin Oncol. 2000, 18:646-50. 10.1200/JCO.2000.18.3.646 